\title{
Commentary: Finding a niche in our TAVR-centric world
}

\author{
Fraser D. Rubens, MD, MSc, FACS, FRCSC
}

Multiple new aortic prostheses have been introduced in the same decade that transaortic valve replacement (TAVR) has exploded. TAVR has reshaped valve practice with a whole new set of priorities, and the Trifecta valve is trying to find its niche in this environment. Its novel design has been associated with a consistent improvement in early hemodynamics compared with other valves, with one-half the incidence of patient- prosthesis mismatch (PPM). Surgeons are excited about the possibilities, and in many institutions, the Trifecta has become the valve of choice for small aortic roots.

However, a nagging question persists as to whether the minor hemodynamic advantages translate into clinical benefit. Furthermore, owing to the structural similarity of another valve that has been associated with premature structural valve disease (SVD), durability remains a concern.

In this issue of the Journal, Lehmann and colleagues ${ }^{1}$ present a large clinical series with the Trifecta valve, presenting medium-term outcome data that to this point are not dissimilar from data reported for other bovine pericardial valves. The data show a low incidence of moderate PPM (5.5\%), with no severe PPM. The clinical outcomes are excellent despite the high incidence of preoperative shock and high morbidity in their referral population.

Most importantly, the authors report the frequency of aortic reintervention in the case of SVD. We cannot confuse this with the actual incidence of SVD, because we do not have follow-up echocardiographic data for each patient. ${ }^{2}$ There is also the possibility that SVD has occurred that has caused clinical deterioration precluding intervention

\footnotetext{
From the Division of Cardiac Surgery, University of Ottawa Heart Institute, Ottawa, Ontario, Canada.

Disclosures: Dr Rubens is a consultant for Abbott Medical.

Received for publication Nov 10, 2019; accepted for publication Nov 12, 2019; available ahead of print Dec 9, 2019.

Address for reprints: Fraser D. Rubens, MD, MSc, FACS, FRCSC, Ottawa Heart Institute, 40 Ruskin St, Room H304a, Ottawa, Ontario, Canada K1Y 4W7 (E-mail: frubens@ottawaheart.ca).

J Thorac Cardiovasc Surg 2021;161:1750-1

$0022-5223 / \$ 36.00$

Copyright (c) 2019 by The American Association for Thoracic Surgery

https://doi.org/10.1016/j.jtcvs.2019.11.078
}

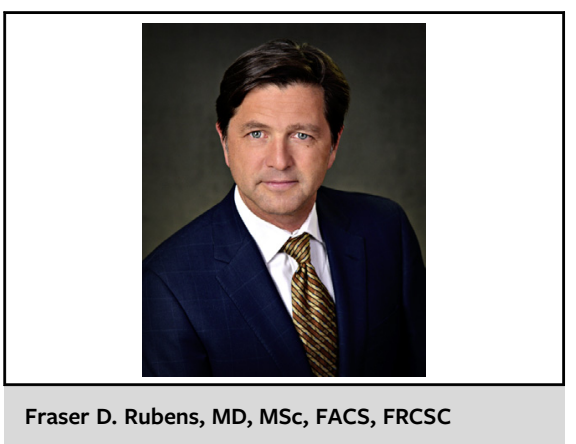

CENTRAL MESSAGE

This series presents results that inspire confidence with mediumterm outcomes and the feasibility of valve-in-valve intervention, cautiously shaping our surgical indications for this valve.

or that is not deemed to warrant surgery at this point despite symptoms, or in cases where the patient is not a surgical candidate or refuses surgery and the anatomy is not favorable for nonsurgical intervention.

This is also one of the largest experiences demonstrating the feasibility of valve-in-valve (VinV) treatment for SVD. Seventeen of 30 patients underwent VinV, with acceptable early results. There were 2 revealing findings in this cohort. In 5 cases, VinV was completed in cases in which the original surgical aortic valve replacement (SAVR) was a size 21. Second, in 1 patient, a VinV was successful despite PPM after the original SAVR. Unfortunately, we do not know why the additional 13 patients were not offered VinV. Was this decision basis on predicted coronary obstruction?

It appears that with this large series, the Trifecta valve has crossed the Rubicon, as based on similar data for the Mitroflow, one would expect to see an $8 \%$ incidence of SVD at this point. ${ }^{3}$ On the other hand, there have been worrisome reports in other case series of premature SVD inconsistent with the current study. ${ }^{4}$ The follow-up here is relatively short, and though we've gone one lap, there are several laps to go. We still don't truly know who benefits the most from this valve, but at least we are slowly narrowing the field.

\section{References}

1. Lehmann S, Jawad K, Dieterlen MT, Hoyer A, Garbade J, Davierwala P, et al. Durability and clinical experience using a bovine pericardial prosthetic aortic valve. J Thorac Cardiovasc Surg. 2021;161:1742-9. 
2. Capodanno D, Petronio AS, Prendergast B, Eltchaninoff H, Vahanian A, Modine T, et al. Standardized definitions of structural deterioration and valve failure in assessing long-term durability of transcatheter and surgical aortic bioprosthetic valves: a consensus statement from the European Association of Percutaneous Cardiovascular Interventions (EAPCI) endorsed by the European Society of Cardiology (ESC) and the European Association for Cardio-Thoracic Surgery (EACTS). Eur Heart J. 2017;38:3382-90.
3. Sénage T, Le Tourneau T, Foucher Y, Pattier S, Cueff C, Michel M, et al Early structural valve deterioration of Mitroflow aortic bioprosthesis: mode, incidence, and impact on outcome in a large cohort of patients. Circulation. 2014; 130:2012-20.

4. Fukuhara S, Shiomi S, Yang B, Kim K, Bolling SF, Haft J, et al. Early structural valve degeneration of trifecta bioprosthesis. Ann Thorac Surg. August 6, 2019 [Epub ahead of print].
See Article page 1742.

\section{Commentary: The valve lasts, until it doesn't: then what?}

\author{
Nels D. Carroll, MD, and Dawn S. Hui, MD
}

In this edition of the Journal, Dr Lehmann and colleagues ${ }^{1}$ present their institutional long-term experience with the Trifecta bioprosthetic valve (Abbott Structural Heart, St Paul, Minn) from 2007 to 2018. Including 1241 patients and spanning 10 years, this represents a substantial cohort for analysis. Although the authors report a relatively high rate of morbidity, including $44.7 \%$ of patients with arrhythmias, $22.9 \%$ with respiratory failure, and $12.9 \%$ renal failure requiring dialysis, they attribute this to a high-risk patient population, with a median EuroSCORE of 7.8. The durability of the valve is supported by an actuarial treatment rate of $1.3 \%$ at 5 years and $6.7 \%$ at 8 years for structural valve deterioration (SVD). The median time to SVD requiring treatment for the Trifecta valve in this study was 5.5 years. This compares well with the reported actuarial explant rate of $1.9 \%$ at 10 years for the Perimount valve (Edwards Lifesciences, Irvine, Calif) and the $0.9 \%$ rate of SVD at 5 years for the newer-generation Magna Ease valve (Edwards Lifesciences)., ${ }^{2,3}$

Of interest is the spectrum of treatments performed for SVD. Comparable studies, such as that cited for the Perimount valve, have not included the option of valve-invalve $(\mathrm{ViV})$ transcatheter intervention (TAVI) for the

\footnotetext{
From the Department of Cardiothoracic Surgery, University of Texas Health Science Center at San Antonio, San Antonio, Tex.

Disclosures: Authors have nothing to disclose with regard to commercial support.

Received for publication Nov 20, 2019; accepted for publication Nov 20, 2019; available ahead of print Dec 9, 2019.

Address for reprints: Dawn S. Hui, MD, 7703 Floyd Curl Drive, Suite 211L, San Antonio, TX 78229 (E-mail: huid@uthscsa.edu).

J Thorac Cardiovasc Surg 2021;161:1751-2

$0022-5223 / \$ 36.00$

Copyright (c) 2019 by The American Association for Thoracic Surgery

https://doi.org/10.1016/j.jtcvs.2019.11.079
}

Check for updates

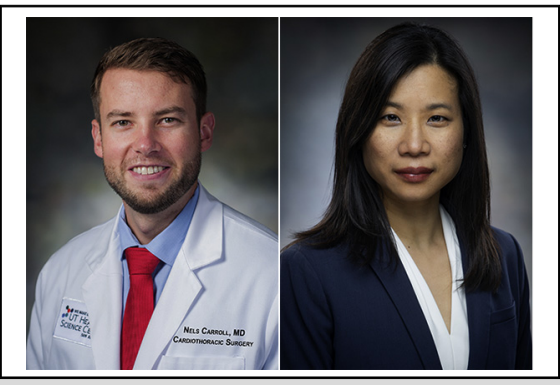

Nels D. Carroll, MD (left), and Dawn S. Hui, MD (right)

\section{CENTRAL MESSAGE \\ Transcatheter interventions in- crease the range of therapeutic possibilities for structural valve degeneration. Contemporary bioprosthetic durability studies should be interpreted with this in mind.}

treatment for SVD. In the current study, 13 of the 30 patients who underwent reintervention for SVD had conventional surgical explant and replacement, whereas 17 underwent ViV TAVI with no cases of coronary obstruction. ViV became more common over the course of this study and reflects the real-world application of a burgeoning technology. ${ }^{4}$

Valve success can be defined in several ways, including SVD by echocardiographic criteria and by the clinical need for reintervention. The former is a truer reflection of durability, but accurate long-term data may be difficult to obtain in real-world practice. The latter is only a proxy for durability and is less accurate, given that some patients may be considered a poor risk for surgery or may elect to not pursue repeat surgery. Before ViV TAVI, the only available reintervention was reoperative aortic valve surgery. ViV 